\title{
Dysphagia in Ramsay Hunt's Syndrome - A Case Report -
}

\author{
Jae Hoon Shim, M.D., Jin-Woo Park, M.D., Bum Sun Kwon, M.D., Ki Hyung Ryu, M.D., Ho Jun Lee, M.D., \\ Woo Hyun Lim, M.D., Jung Hwan Lee, M.D., Young Geun Park, M.D.
}

Department of Physical Medicine and Rehabilitation, College of Medicine, Dongguk University, Goyang 410-773, Korea

Ramsay-Hunt syndrome is caused by varicella zoster virus infection in the geniculate ganglion of the facial nerve. It is characterized by facial palsy, otic pain, and herpetic vesicles around the auricle and external auditory canal. Additionally, symptoms may develop related to other cranial nerve involvement, such as dizziness or hearing loss by the vestibulocochlear nerve being invaded. We report a rare case of a Ramsay-Hunt syndrome patient who developed dysphagia due to multiple cranial nerve involvement including the glossopharyngeal nerve and vagus nerve.

Key Words Herpes zoster oticus, Dysphagia, Facial paralysis

\section{INTRODUCTION}

Ramsay-Hunt's syndrome is also known as geniculate ganglion herpes or otic zoster. Main characteristics include ipsilateral facial palsy and herpes zoster features of the auricle, external auditory canal, and tympanic membrane, and is often accompanied by neurological symptoms of the inner ear. ${ }^{1}$ Cases in which the facial nerve and vestibulocochlear nerve are simultaneously involved are commonly reported, but involvement of other cranial nerves is extremely rare. In this article, we report a case

Received June 7, 2010; Accepted December 10, 2010

Corresponding author: Jin-Woo Park

Department of Physical Medicine and Rehabilitation, Dongguk University Ilsan Hospital, 814, Siksa-dong, Ilsandong-gu, Goyang 410773 , Korea

Tel: +82-31-961-7484, Fax: +82-31-961-7488, E-mail: jinwoo.park.md@ gmail.com

(c) This is an open-access article distributed under the terms of the Creative Commons Attribution Non-Commercial License (http:// creativecommons.org/licenses/by-nc/3.0) which permits unrestricted noncommercial use, distribution, and reproduction in any medium, provided the original work is properly cited.

Copyright $\odot 2011$ by Korean Academy of Rehabilitation Medicine of dysphagia in a patient receiving treatment for Ramsay Hunt's syndrome.

\section{CASE REPORT}

A patient, age of 48 years, suffered from odynophagia and pharyngeal swelling after undergoing dental care for toothache 7 days before visiting our department. Right sided otalgia developed 3 days earlier, and vesicles with discharge into the right auricle and external auditory canal were identified 2 days before the visit. On the day of visit, right sided facial palsy and skin eruption of the right jaw, perioral region, and cheek developed, and the patient was admitted to the Department of Otorhinolaryngology by the emergency room. Past medical history, social history, and family history of the patient revealed no remarkable abnormalities. The patient presented with alert consciousness on admission, but complained of hoarseness, headache, dizziness, and hiccups. On swallowing, the patient reported a residual sensation with throat discomfort and frequent aspiration. Physical examination showed normal vital signs, normal 
motor power, and normal levels of deep tendon reflex in all 4 extremities. Erythematous vesicles and pustules accompanied by pain of the right auricle and external auditory canal developed, and vesicles at the ipsilateral jaw, perioral region, and cheek were also identified. Facial sensory was equivocally normal, but the patient presented right sided lagophthalmos, loss of nasolabial fold, drop of oral angle, and loss of forehead wrinkles (Fig.
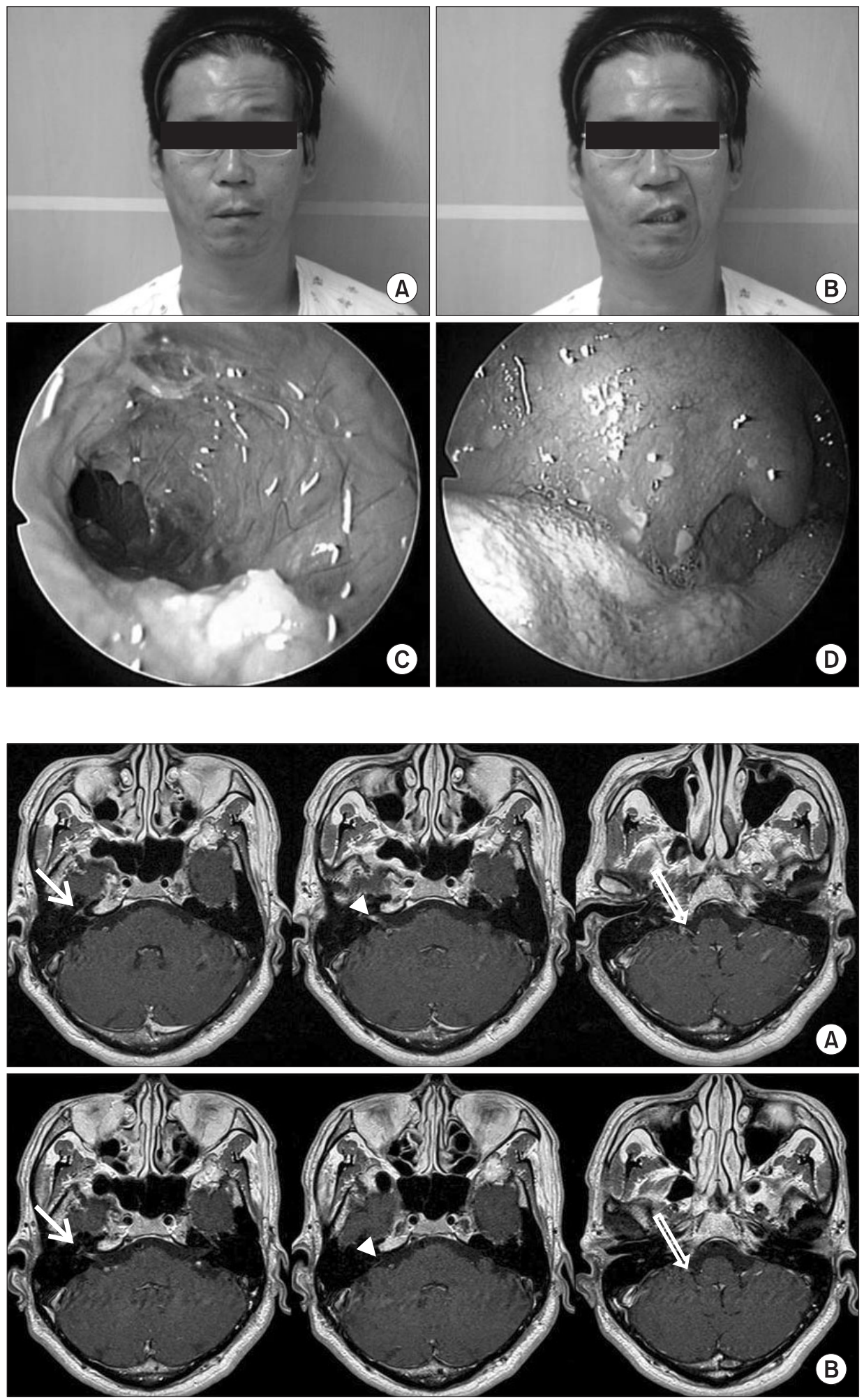

Fig. 1. Clinical features. (A) No forehead motion is observed. (B) An asymmetric mouth is noted with maximal effort. (C) Much discharge is observed in the external auditory canal. (D) Vesicles are noted on the anterior pillar, soft palate, and uvula.

Fig. 2. Contrast internal auditory canal MRI. (A) Contrast-enhanced axial T1-weighted image (onset) shows enhancement in the geniculate ganglion (arrow), cisternal and internal auditory canal segment of facial and vestibulocochlear nerve (arrowhead), and cisternal segment of the glossopharyngeal and vagus nerve (open arrow). (B) Contrastenhanced axial T1-weighted image (2 months after onset) shows decreased enhancement in the geniculate ganglion (arrow), cisternal and internal auditory canal segments of facial and vestibulocochlear nerves (arrowhead), and cisternal segment of the glossopharyngeal and vagus nerves (open arrow). 
1). Neuroconductive studies revealed absence of action potential at the right frontalis, orbicularis oculi, nasalis, and orbicularis oris muscles. Laryngoscopic evaluation showed right vocal cord palsy. Laboratory studies showed WBC 5,270/ul neutrophils $64.3 \%$ which were within normal limits, but a highly sensitive C-reactive protein (hsCRP) level was elevated to $4.434 \mathrm{mg} / \mathrm{dl}$. Blood tests showed positive results for Herpes IgG and VZV IgG, and negative results for Herpes IgM and VZV IgM. A contrast enhanced magnetic resonance imaging study of the inner auditory canal was conducted for differential diagnosis of other diseases. High signal intensity at the basal neural canal of the right inner auditory canal was shown on T1 weighted imaging (Fig. 2-A), and no other abnormal findings such as acute cerebral infarction were identified. Treatment with intravenous administration of acyclovir $(1,800 \mathrm{mg})$ for 2 weeks was carried out along with prednisolone $(60 \mathrm{mg}$ ) for 4 weeks with gradual reduction of dosage. For management of skin lesions and prevention of secondary infection, frequent wet dressings were provided using acyclovir cream and mupirocin ointment. On the second day of admission, the patient was transferred to the Department of Neurology for further evaluation of aggravated dysphagia, hoarseness, dizziness, and hiccups. Brain magnetic resonance imaging studies revealed no remarkable abnormal findings, but CSF examination revealed elevated levels of protein to 70.0 $\mathrm{mg} / \mathrm{dL}$. In addition, a VZV DNA PCR (polymerase chain reaction), which was previously proven positive, showed negative results following administration of intravenous antiviral agents. A video fluoroscopic swallowing test conducted on day 17 of admission showed large amounts of residue distributed asymmetrically on the pyriform sinus and vallecular fossa. Examination after turning the patient's neck towards the right showed decreased amounts of residue (Fig. 3). Thereafter, education concerning adequate positioning and occupational therapy, including functional electrical stimulation treatment for muscle strengthening, was carried out. Normal swallowing was eventually enabled at the time of discharge, but there were no definite improvements in dizziness, facial palsy, and hoarseness. A second trial using contrast enhanced internal auditory canal MRI evaluation was performed 2 months following discharge. MRI findings revealed diminished high signal intensity of the right internal auditory canal, and there were no newly developed lesions (Fig. 2-B).

\section{DISCUSSION}

Ramsay-Hunt's syndrome develops due to infection by herpes zoster virus in the geniculate ganglion of the facial nerve, and leads to facial palsy, otalgia, and skin lesions of the auricle and external auditory canal. In addition, symptoms such as dizziness and hearing loss may develop following involvement of the vestibulocochlear nerve. Rarely, there have been case reports describing simultaneous involvement of the glossopharyngeal nerve and vagus nerve. ${ }^{1}$ This article reports a case of RamsayHunt syndrome in which facial palsy, otalgia, hoarseness, and dizziness developed along with dysphagia, and involvement of the facial nerve, vestibulocochlear nerve, glossopharyngeal nerve, and vagus nerve. Although the mechanism about descending involvement of lower cra-
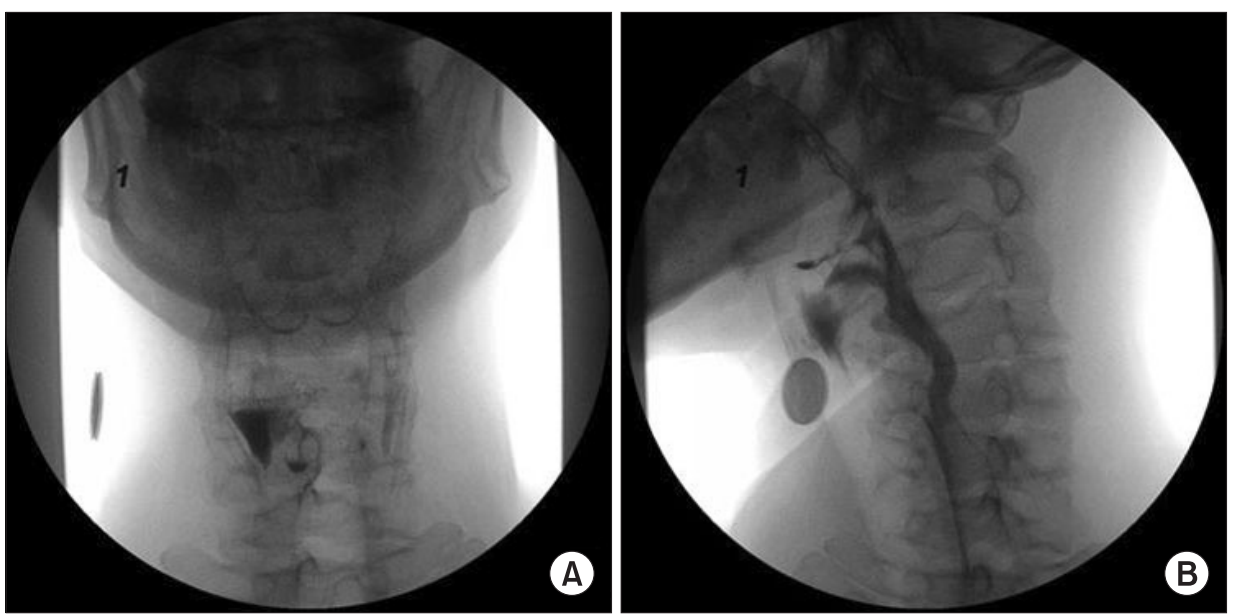

Fig. 3. Video fluoroscopic swallowing study. (A) An anterior radiographic view of the pharynx illustrating residual barium on the right side of the pharynx in the pyriform sinus. (B) An anterior radiographic view of the pharynx with the head turned to the weaker side, illustrating the bolus flow down the opposite side of the pharynx. 
nial nerves is uncertain, several hypotheses have been proposed. First, invasion of cranial nerves may have been due to anatomical locations. The facial nerve joins the vestibulocochlear nerve via anterior and posterior locations of the geniculate ganglion, ${ }^{2}$ and on account of connection with the glossopharyngeal nerve and vagus nerve, ${ }^{3}$ simultaneous viral infection is possible. Secondly, the glossopharyngeal nerve, vagus nerve, accessory nerve and hypoglossal nerve are supplied by the ascending pharyngeal artery. The facial nerve, maxillary nerve, and mandibular nerve of the trigeminal nerve are supplied by the middle meningeal artery. ${ }^{4}$ This supports the possibility of polyneuropathy occurring after vasculitis due to viral infection. ${ }^{5}$ Simultaneous treatment with acyclovir and corticosteroid leads to a better outcome than solitary administration of corticosteroid. ${ }^{6}$ Moreover better prognosis is expected when treatment is initiated within 3 days after occurrence of symptoms. ${ }^{7}$ The prognosis regarding facial palsy is worse compared with Bell's palsy. When acyclovir is administrated, there are no correlations with age or gender, but poor outcomes are reported in cases of deteriorations in hearing or vestibular symptoms. ${ }^{8}$ In complete facial palsy, only $10 \%$ of patients showed improvement. ${ }^{9}$ In this case, treatment with acyclovir and corticosteroid was performed, but the outcomes were unsatisfactory. It is suggested that associated symptoms such as dizziness along with complete facial palsy, and delayed periods of treatment lead to disappointing outcomes. Regarding dysphagia in patients with pharyngeal muscle palsy or deteriorated function of the upper esophageal sphincter, there have been case reports of decreased residue and improved swallowing after positioning the patients' head ipsilaterally. ${ }^{10}$ This study supports the fact that certain maneuvers can improve dysphagia. We report a rare case of dysphagia in Ramsay-Hunt syndrome associated with polyneuropathy.

\section{REFERENCES}

1. Sweeney CJ, Gilden DH. Ramsay Hunt syndrome. J Neurol Neurosurg Psychiatr 2001; 71: 149-154

2. Tschiassny K. Herpes zoster oticus (Ramsay Hunt's syndrome) comments on an article by Johnson and Zonderman. Arch Otolaryngol 1950; 51: 73-82

3. Tananka S, Mizukami S. Vagal communicating branches between the facial and glossopharyngeal nerves, with references to their occurrence from the embryological point of view. Acta Anat (Basel) 1991; 142: 2532

4. Lapresle J, Lasjaunias P. Cranial nerve ischaemic arterial syndromes. A review. Brain 1986; 109: 207-216

5. Kleinschmidt-DeMasters BK, Amlie-Lefond C, Gilden $\mathrm{DH}$. The patterns of varicella zoster virus encephalitis. Human Pathology 1996; 27: 927-938

6. Kinishi M, Amatsu M, Mohri M, Saito M, Hasegawa T, Hasegawa S. Acyclovir improves recovery rate of facial nerve palsy in Ramsay Hunt syndrome. Auris Nasus Larynx 2001; 28: 223-226

7. Murakami S, Hato N, Horiuchi J, Honda N, Gyo K, Yanagihara N. Treatment of Ramsay Hunt syndrome with acyclovir-prednisolone: significance of early diagnosis and treatment. Ann Neurol 1997; 41: 353357

8. Uri N, Greenberg E, Kitzes-Cohen R, Doweck I. Acyclovir in the treatment of Ramsay Hunt syndrome. Otolaryngol Head Neck Surg 2003; 129: 379-381

9. Robillard RB, Hilsinger RL Jr, Adour KK. Ramsay Hunt facial paralysis: clinical analysis of 185 patients. Otolaryngol Head Neck Surg 1986; 95: 292-297

10. Logemann JA, Kahrilas PJ, Kobara M, Vakil NB. The benefit of head rotation on pharyngoesophageal dysphagia. Arch Phys Med Rehabil 1989: 70 :767-771 